\title{
Mapping of QTLs Associated with Seed Vigor to Artificial Aging Using Two RIL Populations in Maize (Zea mays L.)
}

\author{
Zanping Han1, Wang Bin'1, Jun Zhang'2, Shulei Guo², Hengchao Zhang1, \\ Lengrui Xu' ${ }^{1}$, Yanhui Chen ${ }^{2}$ \\ ${ }^{1}$ College of Agronomy, Henan University of Science and Technology, Luoyang, China \\ ${ }^{2}$ College of Agronomy, Henan Agricultural University, Zhengzhou, China \\ Email: hnlyhzp@163.com
}

How to cite this paper: Han, Z.P., Bin, W., Zhang, J., Guo, S.L., Zhang, H.C., Xu, L.R. and Chen, Y.H. (2018) Mapping of QTLs Associated with Seed Vigor to Artificial Aging Using Two RIL Populations in Maize (Zea mays L.). Agricultural Sciences, 9, 397-415.

https://doi.org/10.4236/as.2018.94028

Received: December 20, 2017

Accepted: April 27, 2018

Published: April 30, 2018

Copyright $\odot 2018$ by authors and Scientific Research Publishing Inc. This work is licensed under the Creative Commons Attribution International License (CC BY 4.0).

http://creativecommons.org/licenses/by/4.0/

\begin{abstract}
Improvement in seed vigor under adverse condition is an important object in maize breeding nowadays. Because the higher sowing quality of seeds is necessary for the development of the agriculture production and better able to resist all kinds of adversity in the seeds storage. So it is helpful for long-term preservation of germplasm resource. In our study, two connected recombinant inbred line (RIL) populations, which derived from the crosses Yu82 $\times$ Shen 137 and Yu537A $\times$ Shen 137 respectively, were evaluated for four related traits of seed vigor under three aging treatments. Meta-analysis was used to integrate genetic maps and detected QTL across two populations. In total, 74 QTL and 20 meta-QTL (mQTL) were detected. All QTLs with contributions (R2) over $10 \%$ were consistently detected in at least one of aging treatments and integrated in mQTL. Four key mQTLs (mQTL2-2, mQTL5-3, mQTL6 and mQTL8) with R2 of some initial QTLs > 10\% included 5 - 9 initial QTLs associated with $2-4$ traits. Therefore, the chromosome regions for four mQTLs with high QTL co-localization might be hot spots of the important QTLs for the associated traits. Twenty-two key candidate genes regulating four related traits of seed vigor mapped in 14 corresponding mQTLs. In particular, At5g67360, 45238345/At1g70730/At1g09640 and 298201206 were mapped within the important mQTL5-3, mQTL6 and MQTL8 regions, respectively. Fine mapping or construction of single chromosome segment lines for genetic regions of the three mQTLs is worth further study and could be put to use molecular marker-assisted breeding and pyramiding QTLs in maize.
\end{abstract}

\section{Keywords}

Maize(Zea mays L.), Seed Vigor, RIL, QTL, Artificial Aging 


\section{Introduction}

Seed is consumed as food and animal feed, providing more than $70 \%$ of caloric intake around the world, additionally it is also a fundamental component of the plant life cycle, as they store the genetic information necessary for the next generation of plants to disperse, establish, develop and eventually reproduce to maintain the species [1]. Seed vigor is an important and complex agronomic trait, determined by several factors including genetic and physical purity, mechanical damage and physiological condition, characterized by maintaining a high seed vigor and stable content after storage [2] [3] [4], and required to ensure the rapid and uniform emergence of plants in the field under different environmental conditions. High vigor seeds make a great advantage for growth and production potential, which can enhance germination rates, resistance to environmental stresses, and crop yields [5] [6]. Therefore, farmers and growers are constantly looking for high quality seeds able to ensure uniform germination and growth in field and to increase production.

Seed vigor essentially depends on the ability to withstand prolonged storage and the deleterious effects of aging. Seed vigor during storage can be defined as the maximum time period that pure seeds retain germination viability when stored under ideal environmental conditions and therefore represents an important trait for the conservation of seed resources. It varies among the different species due to natural variability and is usually regarded to be related with seed longevity or seed storability traits [7] [8]. A reliable assay is essential to accurately phenotype the response to seed storability. However, studies of seed longevity under conventional or optimal storage conditions would take years to complete and therefore so-called accelerated aging or controlled deterioration tests (CDT) have been developed to assess the vigor of seed lots and to predict their relative longevity by aging seeds rapidly at elevated temperature and relative humidity (RH) as an alternative to analyze this property more efficiently [9] [10] [11].

Although the environment during seed formation, harvest, and especially storage is important for seed vigor, genetic factors also largely affect seed vigor [12] [13] [14] [15]. Genetics provides a powerful approach such as linkage analysis and, more recently, association mapping for genetic dissection of physiological and molecular bases of phenotypic traits such as seed longevity [16]. The former relies on trait segregation in a population derived from a bi-parental cross, and has been used to identify QTL for seed vigor under conventional storage conditions or CDT in rice, barley, wheat, oilseed rape and model plant Arabidopsis thaliana [7] [13] [14] [17] [18] [19] [20] [21]. The latter is a population-based method that the mapping population consists of a set of unrelated accessions by the detection of linkage disequilibrium between a trait and a genetic marker [22]. However, seed vigor was not reported so far in all species. In addition, proteome analysis of seed vigor in Arabidopsis thaliana, maize revealed common features the CDT or conventionally aged seeds [23] [24] [25]. 
In the present study, 208 and 212 F10 RILs derived from the two crosses between Yu82 and Shen137, Yu537A and Shen137 were used to detect QTL for four traits of seed vigor under control and three aging treatment conditions. The first aim of this research was to identify the QTL traits of seed vigor. The second aim was to integrate QTLs detected across two RIL populations to identify true QTLs, and furthermore was to integrate candidate gene analyses with related traits of seed vigor QTL mapping across two populations to test the effects of numerous candidate genes for the traits known from other species on the natural variations for the traits in maize.

\section{Materials and Methods}

\subsection{Plant Materials and Artificial Aging Treatments}

First, confirm that you have the correct template for your paper size. This template has been tailored for output on the custom paper size $(21 \mathrm{~cm} * 28.5 \mathrm{~cm})$. The two connected populations used in the study consisted of 208 and 212 F10 RILs derived by single-seed descent from two crosses of Yu82 $\times$ Shen137 and Yu537A $\times$ Shen137, which were referred as Population 1 (Pop 1) and Population 2 (Pop 2) and used to identify QTLs for related trait of seed vigor, respectively.

The artificial aging treatment was used the same method described by Zeng et al. [26]. The seeds of two populations and three parents were reproduced in the winter in Hainan Province in 2015. After harvest, the seeds were fully dried under natural conditions. The seeds of each genotype were divided into four portions (60 seeds choosing to ensure sowing quality of every portion) for artificial aging treatments. All the seeds were placed in Nylon mesh belt firstly, then were treated at $45^{\circ} \mathrm{C} \pm 1^{\circ} \mathrm{C}$ and $90 \%$ relative humidity for $0,2,4$, and 6 days (0d, $2 \mathrm{~d}$, $4 \mathrm{~d}$ and $6 \mathrm{~d}$ ) by using a thermostatic moisture regulator, respectively. Every treatment followed a randomized complete block design with three replications. Among treatments, 0d treatment acted as control.

\subsection{Germination Experiment and Related Trait of Seed Vigor Evaluation}

The template is used to format your paper and style the text. All margins, column widths, line spaces, and text fonts are prescribed; please do not alter them. You may note peculiarities. For example, the head margin in this template measures proportionately more than is customary. This measurement and others are deliberate, using specifications that anticipate your paper as one part of the entire journals, and not as an independent document. Please do not revise any of the current designations.

The germination experiment conducted at $25^{\circ} \mathrm{C}$ in artificial climate chamber in 2016. The method of germination experiment was as follows: the first, selecting diameter of $0.05-0.2 \mathrm{~mm}$ of fine sand as sprout bed and the sand was treated by high-handed sterilization pan at $120^{\circ} \mathrm{C}$ for two hours; the second, using a germination container of $16 \times 8$ holes that the diameter of each hole was 40 
$\mathrm{mm}$; the third, each hole was filled with $3.5 \mathrm{~cm}$ thick sand and put 2 seeds in it, and then used $1.5 \mathrm{~cm}$ thick sand to cover them; the last, the germination containers sowing seeds were left in artificial climate chamber for a temperature $25^{\circ} \mathrm{C}$, a relative humidity $65 \%$ and illumination conditions $4000 \mathrm{~lx}$, the photoperiod was 14/10 (day/night). The number of germinated seeds was counted daily. The data of related traits for 8 days after sowing were used for QTL analysis when obvious differences between the parents were observed. After daily statistics finished, 5 plants of each RIL were selected randomly to measure the seedling length, respectively. The germination percentage (GP) was calculated as GP $=\mathrm{n} / \mathrm{N} \times 100 \%$, where $\mathrm{n}$ is the total number of germination seeds, $\mathrm{N}$ is the total number of seeds. The germination index (GI) was calculated as: GI $=\Sigma \mathrm{Gt} / \mathrm{Dt}$, where the Dt is the germination time, Gt is the number of germinated seeds on the time. The vigor index (VI) was calculated as: $\mathrm{VI}=\mathrm{GI} \times \mathrm{SL}$, where the SL is the seedling length on day 8 . The simple vigor index (SVI) was calculated as: SVI $=\mathrm{GP} \times$ SL. The mean germination time $($ MGT) was calculated as: $\mathrm{MGT}=\Sigma \mathrm{Gt} \times$ $\mathrm{Dt} / \mathrm{GP}$, where the sense of Gt and Dt as above.

\subsection{Statistical Analysis of Phenotypic Data}

The trait values for each RIL were reported as the average from five plants in each replication. The overall performance was the average over the three replications from each artificial aging treatment. Analysis of variance (ANOVA) was carried out to estimate genetic variation for all the measured traits among the RILs using the general linear model procedure of the statistical software SPSS 17.0. Descriptive statistics and simple correlation coefficients ( $r$ ) between the traits were calculated using the above statistical software.

\subsection{Construction of Genetic Linkage Map}

A total of 3072 pairs of single nucleotide polymorphism (SNP) markers were selected from the more than 800,000 SNPs to genotype the 420 RILs and three parents. We analyzed polymorphisms of 3072 SNP markers between two pairs of parents, Yu82/Shen137 and Yu537A/Shen137. Ultimately, 1397 and 1371 SNP markers had polyphisms between the two parents, respectively. Chi-square values were generated for 2768 SNP markers, 225 and 232 SNP markers showed serious segregation distortion and failed to be assigned to any linkage in the two populations. The linkage analysis was done with JoinMap version 4.0. Two genetic linkage maps were constructed with 1172 and 1139 SNP markers using Joinmap version 4.0 [27], and the total length $1629.61 \mathrm{cM}$ with an average interval of $1.39 \mathrm{cM}$ for Pop. 1 and $1681.75 \mathrm{cM}$ with an average interval of $1.48 \mathrm{cM}$ for Pop.2 [28].

\subsection{QTL Analysis}

QTL analysis was conducted using composite interval mapping (CIM) with WinQTLcart 2.5 software [29]. For CIM, Model 6 of the Zmapqtl dodule was 
employed for detecting QTL and their effects, specifying the five markers identified by stepwise regression that explained most of the variation for a given trait as forward and backward parameters and a window size of $10 \mathrm{cM}$ on either side of the markers flanking the test site [30]. To identify an accurate significance threshold for each trait, an empirical threshold was determined by performing 1000 random permutations [31]. QTL position was assigned to relevant region at the point of the maximum likelihood odds ratio (LOD). QTL confidence interval was calculated by subtracting one LOD unit on each side from the maximum LOD position [32].

For the additive effects of QTL, positive and negative values indicated that alleles from the normal maize inbred lines Yu82/Yu537A and the maize inbred line Shen 137 increased the trait scores, respectively. QTL were named according to "q" + "artificial aging treatment days" + "trait abbreviation" + "population code" + “-" + "chromosome number" + "QTL number".

\subsection{Meta-QTL Analysis}

To integrate QTLs information for the measured traits located in the two connected RIL populations, the genetic linkage maps were integrated and consensus QTLs were identified by meta-analysis [33] [34]. The QTLs mapped in the two connected RIL populations were projected on the integrated map using their positions and confidence intervals shared by two linkage maps. Some controversial markers between two linkage maps were deleted, which could effectively improve the accuracy of projection.

Meta-analysis was performed by using BioMercator2.1 software [34]. The Akaike Information Criterion (AIC) was used to select the QTL model on each chromosome [35]. According to this, the QTL model with the lowest AIC value is considered a significant model indicating the number of meta-QTL. The number of mQTLs that best fitted the results on a given linkage group was determined based on a modified Akaike criterion [36]. Meta-QTL were named according to "q" + "artificial aging treatment days" + "trait abbreviation" + "population code" + “-" + "chromosome number" + "QTL number".

\section{Results \& Discussion}

\subsection{Phenotypic Performance of Traits Associated with Seed Vigor in Three Parents and Connected Two RILs}

The values of GI, VI and SVI were obviously decreased and the values of MGT were markedly increased after three treatment conditions compared with control in parents and two populations. For three parents, the values of GI, VI and SVI were higher for Yu82 and Yu537A than Shen137 under four aging treatments, while the reverse was true for MGT. trait differences were also found among three parents under each treatment. For RILs, the values presented a large range of variability with transgressive segregation exceeding values of high values par- 
ent. All traits showed normal distribution in the two RIL populations and differed substantially under various treatment conditions (Table 1).

Significant positive correlations were consistently observed for GI, VI and SVI from two RIL populations under control and after various aging treatments except for between VI and SVI from Pop. 1 under 4d aging treatment, while MGT and GI, VI, SVI showed significant negative correlations except for between MGT and SVI from Pop. 2 under 0 and 2d aging treatments (Table 2).

\subsection{QTL Detection for Each Trait in Two Connected Populations}

A total of 74 QTLs for GI, VI, SVI and MGT were detected in two connected populations under control and after three aging treatment conditions, with 40 QTLs in Pop. 1 and 34 QTL in Pop. 2 (Table 3). These QTLs were located on all chromosomes. The contributions to phenotypic variations for a single QTL ranged from $5.33 \%$ to $13.74 \%$, with 10 QTLs over $10 \%$ and 1 QTL over $13 \%$.

\section{GI}

Nine QTLs in Pop.1 and ten QTLs in Pop.2 were identified and located on all chromosomes except for chromosomes 2 and 10 under four aging treatment conditions. The contribution rates of these QTLs ranged from 5.56\% to $13.74 \%$ of total phenotypic variance (Table 3 ). The positive alleles of q2GI1-3, q2GI1-5-1, q2GI1-5-2, q4GI2-4 and q6GI2-9 were derived from Shen137 to contribute towards an increase in values of GI. There were qGI1-6 from Pop.1 consistently mapped in the same marker interval SYN31854-PZE-106102131 after $2 \mathrm{~d}$ and $4 \mathrm{~d}$ aging treatments, qGI2-8-2 from Pop.2 in the interval PZB00865.2-PZE-108073195 after 0d and 2d aging treatments, and qGI2-8-1 from Pop.2 in the interval PZE-105077135-PZE-105082252 after 0d, 2d and 4d aging treatments. Among these QTLs, QTL qGI2-8-1 was responsible for 10.25, 11.73 and $8.22 \%$ of phenotypic variance, and qGI2-8-2 responsible for 10.82 and $7.07 \%$ of phenotypic variance, respectively.

\section{VI}

Eighteen QTLs were mapped for VI under control and after three aging treatments in the two populations, nine in Pop.1 and nine in Pop.2. They were distributed across the whole genome, except for chromosomes 9 and 10 with contribution to phenotypic variation for a single QTL from 5.39 to $8.89 \%$ (Table 3). The positive alleles of qnVI1-1-1, q2VI1-1, q4VI1-1, q4VI21-6 and q6VI1-7 in Pop. 1 and of qnVI2-8 in Pop.2 were contributed by Yu82/Yu537A. However, there was no QTL identified at same marker intervals under different aging treatment conditions in the two populations.

$S V I$

Eleven QTLs for SVI were detected, with four in Pop.1 and seven in Pop.2 under all aging treatment environments. They were distributed across chromosomes 2, 3, 45 and 6, and explained 5.72 to $12.11 \%$ of the phenotypic variation (Table 3). Among these QTLs, two in Pop.1 and one in Pop.2 were derived from Yu82/Yu537A to increase in the trait values. The positive alleles of qSVI2-2 


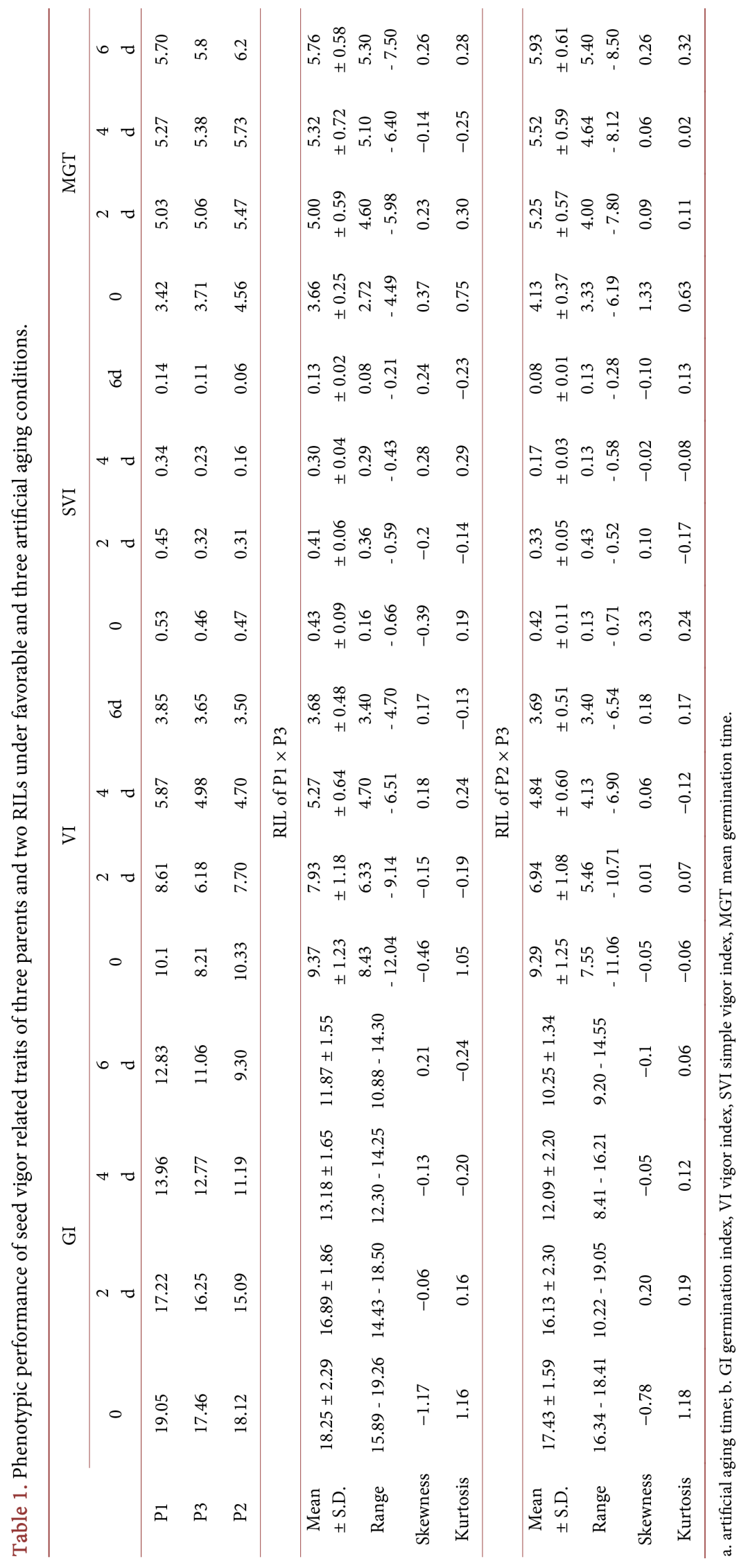


Table 2. Phenotypic correlations among seed vigor related traits for the two RILs based on average under favorable and three artificial aging conditions.

\begin{tabular}{|c|c|c|c|c|c|c|c|c|c|c|}
\hline Treatment $^{\mathrm{a}}$ & Trait $^{\mathrm{b}}$ & GI & VI & SVI & $\operatorname{MGT}(82)$ & & GI & VI & SVI & MGT \\
\hline \multirow[t]{4}{*}{$0 \mathrm{~d}$} & GI & & $0.77^{* *}$ & $0.52^{* *}$ & $-0.18^{*}$ & $2 \mathrm{~d}$ & & $0.85^{* *}$ & $0.66^{* *}$ & $-0.26^{\star *}$ \\
\hline & VI & $0.55^{\star *}$ & & $0.57^{* *}$ & $-0.24^{\star \star}$ & & $0.58^{\star *}$ & & $0.91^{\star *}$ & $-0.34^{\star *}$ \\
\hline & SVI & $0.18^{*}$ & $0.73^{* *}$ & & $-0.20^{*}$ & & $0.44^{* *}$ & $0.95^{\star *}$ & & $-0.35^{\star *}$ \\
\hline & MGT & $-0.64^{* *}$ & $-0.35^{\star *}$ & -0.13 & & & $-0.15^{\star}$ & $-0.17^{\star}$ & -0.08 & \\
\hline \multirow[t]{4}{*}{$4 \mathrm{~d}$} & GI & & $0.34^{* *}$ & $0.37^{\star *}$ & $-0.87^{\star \star}$ & $6 \mathrm{~d}$ & & $0.90^{* *}$ & $0.90^{* *}$ & $-0.42^{\star *}$ \\
\hline & VI & $0.84^{* *}$ & & 0.01 & $-0.41^{\star *}$ & & $0.74^{\star *}$ & & $1.00^{* *}$ & $-0.40^{\star *}$ \\
\hline & SVI & $0.81^{\star *}$ & $0.98^{* *}$ & & $-0.35^{\star *}$ & & $0.68^{\star *}$ & $0.97^{* *}$ & & $-0.34^{* *}$ \\
\hline & MGT & $-0.75^{* *}$ & $-0.59^{* *}$ & $-0.48^{* *}$ & & & $-0.56^{* *}$ & $-0.44^{* *}$ & $-0.26^{* *}$ & \\
\hline
\end{tabular}

The table above diagonal line mean the phenotypic correlations of Yu $82 \times$ Shen137; the table below diagonal line mean the phenotypic correlations of Yu537 $\times$ Shen137. a. artificial aging time; b. GI germination index, VI vigor index, SVI simple vigor index, MGT mean germination time. ${ }^{\star}$ Significant at $\mathrm{P}=0.05$, ${ }^{*}$ Significant at $\mathrm{P}=0.01$.

Table 3. QTL detected for nine traits in the two RIL populations under favorable and three artificial aging conditions.

\begin{tabular}{|c|c|c|c|c|c|c|c|c|}
\hline Trait $^{\mathrm{a}}$ & Treatment & QTL & $\mathrm{Chr}$ & $\begin{array}{c}\text { Position } \\
\text { (cM) }\end{array}$ & Marker Interval & LOD & $\mathrm{R}^{2}(\%)$ & $\mathrm{A}^{\mathrm{b}}$ \\
\hline \multicolumn{9}{|c|}{ Yu $82 \times$ Shen 137} \\
\hline \multirow[t]{9}{*}{ GI } & $\mathrm{N}$ & $q n G I 1-1-1$ & 1 & 89.24 & PZE-101146598-SYN29311 & 3.62 & 7.6 & 0.12 \\
\hline & & $q n G I 1-1-2$ & 1 & 151.75 & PZE-101221874-SYN34116 & 3.39 & 6.74 & 0.08 \\
\hline & $2 \mathrm{~d}$ & $q 2 G I 1-3$ & 3 & 86.05 & PZE-103090188-PZE-103096203 & 3.08 & 5.56 & -0.09 \\
\hline & & $q 2 G I 1-5-1$ & 5 & 73.79 & PZE-105047805-SYN2061 & 2.98 & 6.03 & -0.1 \\
\hline & & $q 2 G I 1-5-2$ & 5 & 175.68 & SYN2910-SYN33425 & 6.78 & 13.74 & -0.15 \\
\hline & & $q 2 G I 1-6$ & 6 & 82.22 & SYN31854-SYN16940 & 3.69 & 6.79 & 0.1 \\
\hline & $4 \mathrm{~d}$ & $q 4 G I 1-1$ & 1 & 157.45 & PZE-101229195-PZE-101229884 & 3.8 & 7.79 & 0.15 \\
\hline & & $q 4 G I 1-6-1$ & 6 & 81.81 & PZE-106097407-PZE-106097584 & 2.69 & 5.72 & 0.12 \\
\hline & & $q 4 G I 1-6-2$ & 6 & 85.82 & SYN16940-PZE-106102131 & 3.69 & 7.59 & 0.14 \\
\hline \multirow[t]{9}{*}{ VI } & $\mathrm{N}$ & $q n V I 1-1-1$ & 1 & 22.53 & PZE-101043600-SYN8490 & 3.22 & 7.06 & 0.06 \\
\hline & & $q n V I 1-1-2$ & 1 & 73.09 & PZE-101129358-PZE-101130082 & 3.38 & 7.42 & -0.08 \\
\hline & $2 \mathrm{~d}$ & $q 2 V I 1-1$ & 1 & 156.85 & PZE-101226516-PZE-101229026 & 2.55 & 5.4 & 0.06 \\
\hline & & $q 2 V I 1-5-1$ & 5 & 164.97 & SYN36222-SYN35254 & 3.88 & 8.41 & -0.08 \\
\hline & & $q 2 V I 1-5-2$ & 5 & 170.56 & SYN14676-SYN14680 & 2.72 & 5.92 & -0.06 \\
\hline & & $q 2 V I 1-5-3$ & 5 & 175.68 & SYN2910-SYN33425 & 3.76 & 8.89 & -0.08 \\
\hline & $4 \mathrm{~d}$ & $q 4 V I 1-1$ & 1 & 160.47 & PZE-101229884-PZE-101232549 & 3.68 & 8.38 & 0.08 \\
\hline & & $q 4 V I 1-6$ & 6 & 85.82 & SYN16940-PZE-106102131 & 2.72 & 5.89 & 0.07 \\
\hline & $6 \mathrm{~d}$ & $q 6 V I 1-7$ & 7 & 108.01 & PZE-107113582-SYN3390 & 3.19 & 7.63 & 0.06 \\
\hline \multirow[t]{4}{*}{ SVI } & $\mathrm{N}$ & $q n S V I 1-5$ & 5 & 175.68 & SYN2910-SYN33425 & 3.76 & 9.29 & -0.03 \\
\hline & $2 \mathrm{~d}$ & $q 2 S V I 1-5$ & 5 & 169.93 & SYN36222-SYN14676 & 3.05 & 6.86 & -0.02 \\
\hline & $4 \mathrm{~d}$ & $q 4 S V I 1-4$ & 4 & 71.8 & PZE-104022145-PZE-104028082 & 3.06 & 7.76 & 0.03 \\
\hline & $6 \mathrm{~d}$ & $q 6 S V I 1-6$ & 6 & 39.06 & PZE-106050123-PZE-106052536 & 2.86 & 6 & 0.02 \\
\hline
\end{tabular}


Z. P. Han et al.

Continued

\begin{tabular}{|c|c|c|c|c|c|c|c|c|}
\hline \multirow[t]{18}{*}{ MGT } & \multirow[t]{5}{*}{$\mathrm{N}$} & $q n M G T 1-2-1$ & 2 & 68.88 & PZE-102062962-SYN24889 & 3.41 & 7.48 & -0.07 \\
\hline & & $q n M G T 1-2-2$ & 2 & 76.04 & PZE-102074262-PZE-102077128 & 4.94 & 10.59 & -0.08 \\
\hline & & $q n M G T 1-2-3$ & 2 & 82.74 & PZE-102080737-PZE-102082146 & 3.23 & 7.06 & -0.07 \\
\hline & & $q n M G T 1-3$ & 3 & 103.93 & PZE-103104806-PZE-103110355 & 3.02 & 7.8 & 0.07 \\
\hline & & $q n M G T 1-6$ & 6 & 73.04 & PZE-106079085-SYN35781 & 2.63 & 5.41 & -0.06 \\
\hline & \multirow[t]{3}{*}{$2 \mathrm{~d}$} & $q 2 M G T 1-3$ & 3 & 113.35 & PZE-103110761-PZE-103115618 & 3.82 & 8.2 & 0.04 \\
\hline & & $q 2 M G T 1-4$ & 4 & 117.11 & PZE-104087575-PZE-104088618 & 2.56 & 5.33 & -0.03 \\
\hline & & $q 2 M G T 1-8$ & 8 & 131.35 & SYN15047-PZE-108133100 & 2.88 & 6.56 & 0.0 .4 \\
\hline & \multirow[t]{4}{*}{$4 \mathrm{~d}$} & $q 4 M G T 1-1$ & 1 & 29.26 & PZE-101056856-SYN13385 & 4.55 & 10.23 & -0.14 \\
\hline & & $q 4 M G T 1-2$ & 2 & 135.94 & PZE-102162330-PZE-102173306 & 2.55 & 6.48 & 0.09 \\
\hline & & $q 4 M G T 1-7$ & 7 & 115.45 & SYN3390-PZE-107126258 & 2.52 & 6.29 & -0.09 \\
\hline & & $q 4 M G T 1-9$ & 9 & 97.31 & PZE-109092637-PZE-109094751 & 2.51 & 5.4 & 0.08 \\
\hline & \multirow[t]{6}{*}{$6 \mathrm{~d}$} & $q 6 M G T 1-4$ & 4 & 182.51 & SYN24017-SYN16139 & 3.09 & 6.63 & 0.11 \\
\hline & & $q 6 M G T 1-5-1$ & 5 & 70.22 & PZE-105044821-PZE-105045328 & 3.16 & 6.99 & 0.13 \\
\hline & & $q 6 M G T 1-5-2$ & 5 & 73.79 & PZE-105047805-SYN2061 & 4.93 & 10.77 & 0.16 \\
\hline & & $q 6 M G T 1-5-3$ & 5 & 76.64 & PZE-105053122-PZE-105053870 & 4.27 & 9.53 & 0.16 \\
\hline & & $q 6 M G T 1-5-4$ & 5 & 87.78 & PZE-105077135-PZE-105082252 & 4.04 & 8.56 & -0.15 \\
\hline & & $q 6 M G T 1-5-5$ & 5 & 90.7 & PZE-105080632-PZE-105093615 & 3.47 & 7.44 & -0.14 \\
\hline \multicolumn{9}{|c|}{ Yu537A × Shen 137} \\
\hline \multirow[t]{10}{*}{ GI } & \multirow[t]{3}{*}{$\mathrm{N}$} & $q n G I-8-1$ & 8 & 76.71 & PZE-108067511-PZE-108069726 & 4.77 & 10.25 & 0.09 \\
\hline & & $q n G I 2-8-2$ & 8 & 82.42 & PZB00865.2-PZE-108073195 & 5.08 & 10.82 & 0.1 \\
\hline & & $q n G I 2-8-3$ & 8 & 92.08 & PZE-108086867-PZE-108087618 & 3.68 & 8.09 & 0.08 \\
\hline & \multirow[t]{2}{*}{$2 \mathrm{~d}$} & $q 2 G I-8-1$ & 8 & 77.71 & PZE-108067511-PZE-108069726 & 5.26 & 11.73 & 0.11 \\
\hline & & $q 2 G \cap-8-2$ & 8 & 83.32 & PZB00865.2-PZE-108073195 & 3.16 & 7.07 & 0.09 \\
\hline & \multirow[t]{2}{*}{$4 \mathrm{~d}$} & $q 4 G I-4$ & 4 & 61.6 & PZE-104035115-PZE-104035657 & 3.82 & 7.81 & -0.11 \\
\hline & & $q 4 G L-8$ & 8 & 76.71 & PZE-108067511-PZE-108069726 & 3.84 & 8.22 & 0.12 \\
\hline & \multirow[t]{3}{*}{$6 \mathrm{~d}$} & $q 6 G I-8$ & 8 & 66.21 & PZE-108059570-PZE-108060445 & 3.52 & 8.33 & 0.11 \\
\hline & & $q 6 G / 2-9$ & 9 & 60.07 & SYN34709-PZE-109061773 & 2.73 & 6.17 & -0.09 \\
\hline & & $q 6 G I 2-10$ & 10 & 86.05 & PZE-110040719-PZE-110043433 & 3.07 & 6.45 & 0.1 \\
\hline \multirow[t]{9}{*}{ VI } & \multirow[t]{5}{*}{$\mathrm{N}$} & $q n V I 2-3-1$ & 3 & 111.57 & SYN28063-PZE-103180642 & 3.68 & 7.78 & -0.09 \\
\hline & & $q n V I 2-3-2$ & 3 & 128.76 & PZE-103151399-SYN1576 & 3.71 & 7.59 & -0.1 \\
\hline & & $q n V I 2-4$ & 4 & 81.67 & PZE-104065092-PZE-104067512 & 2.67 & 6.66 & -0.08 \\
\hline & & $q n V I 2-5$ & 5 & 71.5 & PZE-105084712-PZE-105098349 & 2.51 & 5.39 & -0.07 \\
\hline & & $q n V I 2-8$ & 8 & 82.42 & PZB00865.2-PZE-108073195 & 2.81 & 5.41 & 0.55 \\
\hline & \multirow[t]{2}{*}{$2 \mathrm{~d}$} & $q 2 V I 2-2$ & 2 & 98.2 & SYN8399-PZE-102122951 & 2.67 & 6.29 & -0.07 \\
\hline & & $q 2 V I 2-3$ & 3 & 157.75 & PZE-103118406-SYN31220 & 3.84 & 8.86 & -0.09 \\
\hline & $4 \mathrm{~d}$ & $q 4 V I 2-2$ & 2 & 97.2 & SYN8399-PZE-102122951 & 3.39 & 8.19 & -0.08 \\
\hline & $6 d$ & $q 6 V I 2-5$ & 5 & 75.65 & PZE-105100269-PZE-105101905 & 3.58 & 8.66 & -0.07 \\
\hline \multirow{2}{*}{ SVI } & $\mathrm{N}$ & $q n S V I 2-4$ & 4 & 153.8 & PZE-104106033-PZE-104106790 & 2.92 & 6.84 & 0.03 \\
\hline & $2 \mathrm{~d}$ & $q 2 S V R-2$ & 2 & 98.2 & SYN8399-PZE-102122951 & 4.7 & 12.11 & -0.03 \\
\hline
\end{tabular}




\section{Continued}

\begin{tabular}{|c|c|c|c|c|c|c|c|c|}
\hline & & $q 2 S V I-3$ & 3 & 147.58 & SYN23237-PZE-103139833 & 3.4 & 8.07 & -0.03 \\
\hline & $4 \mathrm{~d}$ & $q 4 S V I-2$ & 2 & 98.2 & SYN8399-PZE-102122951 & 4.31 & 10.67 & -0.03 \\
\hline & & $q 4 S V I 2-3$ & 3 & 126.76 & SYN37386-PZE-103151399 & 2.57 & 5.72 & -0.02 \\
\hline & $6 \mathrm{~d}$ & $q 6 S V I 2-4$ & 4 & 179.96 & PZE-104118950-PUT-60354034273 & 3.37 & 7.92 & -0.02 \\
\hline \multirow[t]{9}{*}{ MGT } & $\mathrm{N}$ & $q n M G T 2-2$ & 2 & 116.59 & PZE-102131962-SYN15147 & 2.69 & 5.9 & -0.09 \\
\hline & & $q n M G T 2-4$ & 4 & 163.63 & PZE-104109431-PZE-104113905 & 2.79 & 6.54 & 0.11 \\
\hline & & $q n M G T 2-5$ & 5 & 109.47 & PZA02629.16-PZE-105128434 & 3.81 & 8.34 & -0.11 \\
\hline & & $q n M G T 2-8$ & 8 & 82.42 & PZB00865.2-PZE-108073195 & 3.81 & 8.46 & -0.11 \\
\hline & $2 \mathrm{~d}$ & $q 2 M G T 2-1$ & 1 & 111.8 & PZE-101140869-PZE-101144216 & 2.84 & 6.39 & -0.11 \\
\hline & & $q 2 M G T 2-2$ & 2 & 116.59 & PZE-102131962-PZE-102136708 & 3.88 & 8.35 & -0.13 \\
\hline & & $q 2 M G T 2-5$ & 5 & 109.47 & PZA02629.16-PZE-105128434 & 5.04 & 11.33 & -0.18 \\
\hline & $4 \mathrm{~d}$ & $q 4 M G T 2-2$ & 2 & 85.86 & PZE-102112161-SYN13599 & 2.8 & 6.08 & 0.12 \\
\hline & $6 \mathrm{~d}$ & $q 6 M G T 2-4$ & 4 & 146.2 & PZE-104103557-SYN5704 & 3.61 & 8.22 & 0.19 \\
\hline
\end{tabular}

a. GI germination index, VI vigor index, SVI simple vigor index, MGT mean germination time; b. A is represent for additive effect.

derived from Shen 137 in Pop.2 were consistently identified at the marker interval SYN8399-PZE-102122951 after 2d and 6d aging treatment, respectively. And QTL qSVI2-2 accounted for $12.11 \%$ and $10.67 \%$ of phenotypic variance, respectively.

\section{$M G T$}

Under control and after three aging treatments, nineteen and nine QTLs for MGT were detected on all chromosomes except for chromosome 10 in Pop.1 and Pop. 2 respectively, which explaining from $5.33 \%$ to $11.33 \%$ of the phenotypic variation (Table 3). The all alleles except for eleven QTL in Pop1 and of six in Pop. 2 were derived from Shen 137 to increase in the trait values. qMGT2-2 and q MGT2-5 in Pop.2 were consistently detected at the same marker interval PZE-102131962-SYN15147 and PZE-102131962-PZE-102136708 under control and after $2 \mathrm{~d}$ aging treatment, respectively.

Component heads identify the different components of your paper and are not topically subordinate to each other. Examples include Acknowledgements and References and, for these, the correct style to use is "Heading 5". Use "figure caption" for your Figure captions, and "table head" for your table title. Run-in heads, such as "Abstract", will require you to apply a style (in this case, non-italic) in addition to the style provided by the drop down menu to differentiate the head from the text.

Text heads organize the topics on a relational, hierarchical basis. For example, the paper title is the primary text head because all subsequent material relates and elaborates on this one topic. If there are two or more sub-topics, the next level head should be used and, conversely, if there are not at least two sub-topics, then no subheads should be introduced. Styles named "Heading 1", "Heading 2", 
"Heading 3", and "Heading 4" are prescribed.

\subsection{Genetic Map Integration and MQTL Analysis from the Experimental Results}

The consensus genetic map included 1712 SNP makers and was $1712.6 \mathrm{cM}$ long with an average of $1.00 \mathrm{cM}$ between markers on the basis of two connected populations. Meta-analysis identified mQTL that was associated with the variation of multiple traits measured. So $20 \mathrm{mQTL}$ were detected from the seventy-four initial mapped QTLs in the two RIL populations for four traits measured by using meta-analysis (Table 4). Seventy-two initial QTLs (97.3\%) were integrated in these regions. The $20 \mathrm{mQTLs}$ were located on all chromosomes except for 10 , four on chromosome 4, three on chromosomes 1, 3 and 5, two on chromosome 2, and one on chromosomes 6, 7, 8 and 9. On average, one mQTL included 3.68 initial QTLs with a range from two to nine for 1-3 traits. It is worth noting that all initial QTLs with R2 > 10\% were integrated in 7 mQTLs: mQTL1-1, mQTL2-1, mQTL2-2, mQTL5-1, mQTL5-3, mQTL5-4 and mQTL8.

Initial QTLs included in mQTL8 were all detected for GI under control and after three aging treatments, that in mQTL2-2 for three traits, that mQTL3-2 for two traits and those in mQTL1-3, mQTL3-1, mQTL4-4, mQTL5-1, mQTL5-3, mQTL5-4 for one traits under two conditions, respectively, and those in the rest of the mQTLs for two traits under one condition.

\section{Discussion}

Seed vigor depends on their physiological and genetic conservation potential and on conditions encountered during storage [37] [38]. Seed vigor strongly influences on plant stand establishment, which challenge crop breeders to produce high quality seeds for stabilizing crop yield. A key to achieving the challenge associated with seed vigor is elucidation of the molecular mechanisms underlying the traits. However, there are few papers about the research of the molecular mechanisms associated with the traits in maize. In this study, two sets of connected RIL populations were evaluated for four traits of seed vigor under control and three aging treatment conditions. Seventy-four QTLs were located in total, with 40 QTLs in Pop.1 and 34 in Pop.2. Individual QTL explained from 5.33 to $13.74 \%$ of the traits associated with seed vigor, with 11 QTLs over $10 \%$. Seventy-two initial QTLs were integrated in 20 meta-QTLs (mQTL) using meta-analysis and all initial QTLs with contributions (R2) $>10 \%$ were integrated in mQTLs. Twenty candidate genes for seed vigor were consistently mapped in 13 corresponding mQTLs regions and mainly involved in glycolytic pathway and protein metabolism. These QTLs could provide useful information for marker-assisted selection in improving performance of seed vigor. At the same time, the results had important reference for the fine mapping of the major QTLs and validation of the potential candidate genes, and analyzing the molecular mechanism of seed vigor in maize. 
Table 4. mQTLs for nine traits in the two connected RIL populations under favorable and three artificial aging conditions.

\begin{tabular}{|c|c|c|c|c|c|c|c|c|}
\hline mQTL & $\mathrm{Chr}$ & $\begin{array}{l}\text { Posti-on } \\
\text { (cM) }\end{array}$ & $\begin{array}{l}\text { Confidence } \\
\text { interval(cM) }\end{array}$ & Flanking marker & $\begin{array}{c}\text { Physical } \\
\text { interval }(\mathrm{Mb})\end{array}$ & $\begin{array}{l}\text { No. of } \\
\text { QTL }\end{array}$ & Integrated QTL & Candidate genes \\
\hline mQTL1-1 & 1 & 36.23 & $23.93-48.6$ & $\begin{array}{l}\text { PZE-101043682 } \\
\text {-PZE-101065758 }\end{array}$ & $\begin{array}{l}29889332 \\
-48812512\end{array}$ & 2 & $q n V I 1-1-1 ; q 4 M G T 1-1$ & 226532762 \\
\hline mQTL1-2 & 1 & 119.25 & $106.28-132.21$ & $\begin{array}{l}\text { SYN24128 } \\
\text {-SYN34477 }\end{array}$ & $\begin{array}{c}179459862 \\
-200303983\end{array}$ & 3 & $\begin{array}{c}q n G I 1-1-1 ; q n V I 1-1-2 ; \\
q 2 M G T 2-1\end{array}$ & 224061823 \\
\hline mQTL1-3 & 1 & 236.11 & $219.12-253.09$ & $\begin{array}{l}\text { PZE-101215138 } \\
\text {-PZE-101240161 }\end{array}$ & $\begin{array}{c}265654966 \\
-286060513\end{array}$ & 4 & $\begin{array}{c}q 4 G I 1-1 ; q 4 V I 1-1 ; \\
q 2 V I 1-1 ; q n G I 1-1-2\end{array}$ & 226494943 \\
\hline mQTL2-1 & 2 & 74.14 & $64.7-83.58$ & $\begin{array}{l}\text { PZE-102055831 } \\
\text {-SYN35922 }\end{array}$ & $\begin{array}{c}33590242 \\
-144073650\end{array}$ & 4 & $\begin{array}{c}q 4 M G T 2-2 ; q 4 M G T 1-2-3 \\
q n M G T 1-2-2 ; q n M G T 1-2-1\end{array}$ & $\begin{array}{l}305671643 ; \\
22284\end{array}$ \\
\hline mQTL2-2 & 2 & 99.72 & $94.5-104.93$ & $\begin{array}{l}\text { PZE-102119932 } \\
\text {-SYN34721 }\end{array}$ & $\begin{array}{c}160591358 \\
-174854687\end{array}$ & 6 & $\begin{array}{c}q n M G T 2-2 ; q 2 M G T 2-2 ; \\
q 2 V I 2-2 ; q 4 V I 2-2 ; q 2 S V I 2-2 ; \\
q 4 S V I 2-2\end{array}$ & \\
\hline mQTL3-1 & 3 & 68.59 & $64.5-72.69$ & $\begin{array}{l}\text { SYN36772 } \\
\text {-SYN37724 }\end{array}$ & $\begin{array}{l}13312517 \\
-25865416\end{array}$ & 3 & $\begin{array}{c}q 2 M G T 1-3-1 ; q n M G T 1-3 \\
q 2 G I 1-3\end{array}$ & $\begin{array}{l}242056533 ; \\
195605946 ; \\
162459222\end{array}$ \\
\hline mQTL3-2 & 3 & 120.19 & $111.3-129.01$ & $\begin{array}{l}\text { SYN37386 } \\
\text {-SYN28063 }\end{array}$ & $\begin{array}{c}194157875 \\
-207200950\end{array}$ & 5 & $\begin{array}{c}q n V I-3-1 ; q n V I 2-3-2 ; \\
q n M G T 1-3 ; q 2 M G T 1-3-1 ; \\
q 4 S V R-3\end{array}$ & $\begin{array}{l}\text { 162459414; } \\
\text { At5g19550 }\end{array}$ \\
\hline mQTL3-3 & 3 & 157.66 & $149.74-165.58$ & $\begin{array}{l}\text { PZE-103115618 } \\
\text {-SYN20493 }\end{array}$ & $\begin{array}{c}175554472 \\
-184720973\end{array}$ & 2 & $q 2 S V I 2-3 ; q 2 V I 2-3$ & At5g51440 \\
\hline mQTL4-1 & 4 & 57.61 & $45.2-70.02$ & $\begin{array}{l}\text { PZE-104050909 } \\
\text {-PZE-104045752 }\end{array}$ & $\begin{array}{c}68235181 \\
-79841743\end{array}$ & 2 & $q 4 S V I 1-4 ; q 4 G I 2-4$ & \\
\hline mQTL4-2 & 4 & 94.31 & $82.2-106.42$ & $\begin{array}{l}\text { PZE-104066884 } \\
\text {-PZE-104087575 }\end{array}$ & $\begin{array}{c}132214044 \\
-162274823\end{array}$ & 2 & $q n V I-4 ; q 2 M G T 1-4$ & $\begin{array}{l}195658029 ; \\
226508796\end{array}$ \\
\hline mQTL4-4 & 4 & 156.64 & $148.52-164.75$ & $\begin{array}{c}\text { PZA02194.1 } \\
- \text { PZE-104115259 }\end{array}$ & $\begin{array}{c}180309373 \\
-196676820\end{array}$ & 3 & $\begin{array}{c}q n S V I 2-4 ; q n M G T 2-4 \\
q 6 M G T 2-4\end{array}$ & \\
\hline mQTL4-3 & 4 & 186.52 & $175.22-197.82$ & $\begin{array}{c}\text { SYN18852 } \\
\text {-PZE-104157368 }\end{array}$ & $\begin{array}{c}225732497 \\
-240245730\end{array}$ & 2 & $q 6 M G T 1-4 ; q 6 S V I 2-4$ & $\begin{array}{l}326509331 \\
\text { Atlg57720 }\end{array}$ \\
\hline mQTL5-1 & 5 & 43.53 & $33.62-53.44$ & $\begin{array}{l}\text { PZE-105032165 } \\
\text {-SYN6475 }\end{array}$ & $\begin{array}{l}17380699 \\
-59293558\end{array}$ & 4 & $\begin{array}{c}q 2 G I 1-5-1 ; q 6 M G T 1-5-2 ; \\
q 6 M G T 1-5-1 ; \\
q 6 M G T 1-5-3\end{array}$ & 197132370 \\
\hline mQTL5-2 & 5 & 76.35 & $66.14-86.56$ & $\begin{array}{l}\text { PZE-105075207 } \\
\text {-PZE-105111462 }\end{array}$ & $\begin{array}{c}82955415 \\
-168450026\end{array}$ & 4 & $\begin{array}{c}q 6 V D-5 ; q n V R-5 ; \\
q 6 M G T 1-5-5, q 6 M G T 1-5-4\end{array}$ & \\
\hline mQTL5-2 & 5 & 109.74 & $107.45-111.32$ & $\begin{array}{l}\text { PZE-105128434 } \\
\text {-PZA02629.16 }\end{array}$ & $\begin{array}{c}184820554 \\
-183705562\end{array}$ & 2 & $q n M G T 2-5 ; q 2 M G T 2-5$ & \\
\hline mQTL5-3 & 5 & 140.93 & $136.83-145.02$ & $\begin{array}{c}\text { SYN36222 } \\
\text {-PZE-105179864 }\end{array}$ & $\begin{array}{c}211582817 \\
-214427178\end{array}$ & 6 & $\begin{array}{l}q n S V I 1-5-2 ; q 2 V I 1-5-1 ; \\
q 2 V I 1-5-2 ; q 2 G I 1-5-2 ; \\
q 2 S V I 1-5 ; q 2 V I 1-5-3\end{array}$ & At5g67360 \\
\hline mQTL6 & 6 & 113.98 & $106.4-121.56$ & $\begin{array}{l}\text { PZE-106083335 } \\
- \text { PZE-106105801 }\end{array}$ & $\begin{array}{c}140906714 \\
-156368157\end{array}$ & 5 & $\begin{array}{c}q n M G T 1-6 ; q 2 G I 1-6 ; \\
q 4 G I 1-6-1 ; q 4 V I 1-6 ; \\
q 4 G I 1-6-2\end{array}$ & $\begin{array}{l}45238345 ; \\
\text { Atlg70730; } \\
\text { At1g09640 }\end{array}$ \\
\hline mQTL7 & 7 & 132.15 & $114.47-149.82$ & $\begin{array}{l}\text { PZE-107116723 } \\
\text {-PZE-107135859 }\end{array}$ & $\begin{array}{c}164071283 \\
-174102543\end{array}$ & 2 & $q 4 G M T 1-7 ; q 6 V I 1-7$ & 146325682 \\
\hline mQTL8 & 8 & 79.5 & $73.49-85.52$ & $\begin{array}{l}\text { ZM012274-0351 } \\
-P Z E-108080736\end{array}$ & $\begin{array}{c}116211524 \\
-135822188\end{array}$ & 9 & $\begin{array}{c}q n G D-8-1 ; q n G D-8-2 ; \\
q n G D 2-8-3 ; q n V I 2-8 ; \\
q n M G T 2-8 ; q 2 G D-8-2 ; \\
q 2 G D-8-1 ; q 4 G D-8 ; \\
q 6 G D-8\end{array}$ & 298201206 \\
\hline mQTL9 & 9 & 65.41 & $58.58-72.23$ & $\begin{array}{l}\text { PZE-109057210 } \\
\text {-SYN18127 }\end{array}$ & $\begin{array}{c}98356545 \\
-114176736\end{array}$ & 2 & $q 4 M G T 1-9 ; q 6 G I 2-9$ & \\
\hline
\end{tabular}




\subsection{Comparison of QTL Located and Synthesis of Initial QTL in the Two Connected RIL Populations}

Comparing the results in both populations, the research found that two common QTLs were located in the same chromosome regions, one for GI at bin 1.09 and the other for MGT at bin 10.03; two common QTLs were located near chromosome regions, one for VI at bin $4.08-4.09$ and the other for SVI at bin 4.08 4.09 , respectively. These QTLs showed great consistency across both populations, which might deserve further study in molecular marker-assisted selection (MAS). Although the two populations exhibited certain similarity because they share one of the parental lines, population-specific QTLs were also found. Three QTLs on chromosomes 9 and 10 were detected in Pop1 under 1 - 2 treatments. These QTLs were contributed by the unique parental line Yu82. One factor of inconsistency of QTL was the use of different bi-parent populations. Difference in the genetic background between the two populations exists because they share only one common parental line.

Twenty mQTLs were detected from the total of 74 initial QTLs for the traits measured using mQTL analysis proposed by Goffinet and Gerber [36] in this study, containing all QTLs of R2 over 10\%. Genomic regions for the traits were mainly focused on chromosomes 1, 3, 4 and 5, even though the mQTLs were mapped on all chromosomes except for chromosome 10. Among the mQTLs, mQTL2-2, mQTL5-4 and mQTL8 with a major effect (R2 of initial QTL > 10\%) included in 6 - 9 initial QTLs for 1 - 3 traits under 2 - 4 aging treatment conditions in one population or both populations. So the genomic regions might be hot spots of the important QTLs for the traits. Fine mapping of the mQTLs and validation of the potential candidate genes were a reliable and feasible strategy for QTL cloning. Therefore, Near-isogenic lines for three mQTLs are now in construction using marker-assisted selection, and the mQTLs might be used to improve maize seed vigor in the near future.

\subsection{Associations between QTL and Candidate Genes in Maize}

Maize is grown widely throughout the world in a range of agro-ecological environments. Companies sell many different registered hybrids in the same ecological region because of the high profits from producing hybrid maize. The amount of hybrid seeds produced by a few large companies could be excess to the requirements of market, so that the extra seeds stored to sell the following year. In the process of seed storage, the aspects of adverse factors associated with high temperature, high moisture content, and high oxygen gas pressure would induce free radical-mediated lipid peroxidation, enzyme inactivation, protein degradation, disruption of cellular membranes, and damage to genetic (nucleic acids) integrity [39] [40] [41]. Therefore, they could accelerate seed deterioration, and decrease seed quality and vigor. To understand further the genetic basis of seed vigor variation during seed storage, the association between QTLs and genes known to be involved in seed vigor in Arabidopsis [38] and maize [42] 
were investigated through a bioinformatics approach in maize. Twenty-two candidate genes for seed vigor were mapped in $15 \mathrm{mQTL}$ intervals (Table 4). The candidate genes were involved in response to stress, molecular chaperones, hydrolase, energy, cell growth/division, protein destination and storage, signal transduction, translation and protein metabolism, amino acid metabolism and other processes.

\subsection{Protein Metabolism Is Major Components of Seed Vigor during Seed Accelerated Aging}

Seed germinations had requirement for some protein metabolisms regrouped several functions during seed accelerated aging, containing protein translocation, folding, thermotolerance, oligomeric assembly, and switching between active and inactive protein conformations [38]. The simultaneous impairment of these functions was closely linked with the loss of seed vigor [38]. Ten candidate genes identified within corresponding mQTLs detected under aging treatments were related to the protein metabolism in this study. 226494943 (Glutathione S-transferase) for defence was associated with the mQTL1-3 region affecting GI and VI; 146325682 (thioredoxin peroxidase) corresponded to the mQTL7 region for VI and MET. These gene families are major antioxidant enzymes in plant cells, as they were significantly accumulated in germinating seeds [43] [44], suggesting the antioxidant enzymes play an important role in seed viability maintenance. Plenty of works also revealed that the activities of anti-oxidases (like SOD, CAT, APX) and the content of antioxidant (like L-ascorbic acid and glutathione) changed upon aging treatment in seeds of soybean, rice, sunflower, maize etc. AT1G57720 (elongation factor 1-g2) for protein metabolism was mapped to the mQTL4-3 interval affecting SVI and MGT. AT1G09640 (Elongation factor 1B-g) for translation and protein metabolism corresponded to the same mQTL6-1 interval associated with GI, VI and MGT. At5g19550 (Asp aminotransferase) for protein metabolism was mapped to the same mQTL3-2 interval for VI, SVI and MGT. 242056533 (Hypothetical ACD_ScHsp26_like), 195605946 (HSP16.9) and 162459222 (HSP17.2) for molecular chaperone were located in the same mQTL3-1 region affecting GI and MGT; At5g51440 (hsp20/alpha crystallin family protein) for molecular chaperone was mapped to the mQTL3-3 interval associated with VI and SVI. The candidate genes (Hsp26, hsp20, HSP16.9 and HSP17.2) with chaperone activities in aged maize seeds might disturb signal transduction such as in responses to stresses like heat shock [45] [46] and also favored targets for oxidation, presumably because they act as shields protecting other proteins against ROS damage [47]. 298201206 (Stress-related protein) was located in the mQTL8 interval for GI, VI and MGT. It has specific role in the detoxication of a wide range of exogenous and endogenous toxicants in artificially aged maize seeds [42].

In addition, three candidate genes identified within mQTL detected under aging treatments were associated with the storage protein and protease in this 
study. 22284 (Vicilin-like embryo storage protein) were mapped to the same mQTL3-3 region associated with VI, SVI and MGT; 226508796 (CAAX prenyl protease 1) for hydrolase were associated with the mQTL4-2 interval affecting VI and MGT; At5g67360 (Cucumisin-like Ser protease) for protease corresponded with the mQTL5-3 region for MGT. The results showed artificial aging would increase proteases and breake down stored proteins, impaired metabolism and energy supply, and ultimately resulted in seed deterioration [48] [49].

\subsection{The Glycolytic Pathway Is Affected during Seed Accelerated Aging}

In this study, six candidate genes identified within mQTL detected under aging treatments were associated with the glycolytic pathway. 226532762 (glyoxalase family protein) in responses to stresses were mapped in the MQTL1-1 region affecting VI and MGT; 45238345 (Aldehyde dehydrogenase) involved in response to stress and Atlg70730 (Phosphoglucomutase) for energy were located in the mQTL6 interval connected with GI, VI and MGT; 326509331 (V-type $\left(\mathrm{H}^{+}\right)$-ATPase domain) for energy was mapped in the same mQTL4-4 interval associated with SVI and MGT; 305671643 (ATP synthase beta subunit) and 197132370 (ATP synthase CF1 beta subunit) associated with energy were located in the mQTL2-1 and mQTL5-1 regions, respectively. The key enzymes/proteins participated in glycolysis, TCA cycle, electron transport chain and oxidative phosphorylation glycolysis by seed aging, which played a major role in the maintenance of the intracellular redoxstate and the maintenance of seed vigor [50]. The results showed that seeds experienced an oxidative stress during aging treatments and mounted a protective response through modification of the glycolytic pathway [51] [52] [53]. We believe that the candidate genes participate in metabolism and energy supply play important roles in seed aging and seed vigor.

\subsection{Other Pathways Were Involved in Seed Vigor during Seed Accelerated Aging}

In this way, other pathways were also involved in seed vigor after accelerated aging except for the above two pathways. For example, the embryo cell undergoes active division and expansion during seed germination. The events might be affected by accelerated aging treatment, as seeds germinated at a much lower speed after aging [54]. In our study, 224061823 (Predicted cyclin-dependent kinase A, CDK) for cell growth/division was mapped in the same mQTL1-3 interval associated with GI and VI; 162459414 (MEK homolog1) for cell growth/division were mapped to the same mQTL3-2 interval associated with VI, MGT and SVI, In addition, 195658029 (Lipoprotein) for lipid metabolism was mapped to the same mQTL4-2 interval associated with VI and MGT. CDK and MEK homolog 1 played a pivotal role in the regulation of the eukaryotic cell cycle. 


\section{Conclusion}

In conclusion, the consistency of the QTL and the candidate genes identified in this study provided valuable information for further finding quantitative trait genes. The alleles for seed vigor could be useful targets for marker-assisted selection to produce germplasm of aging resistance.

\section{Acknowledgements}

This work was supported by grants from the National Natural Science Foundation of China (No.U1504315), Corn Industry Technology System in Henan Province (No.S2010-02) and the Scientific Research Foundation of Henan University of Science and Technology (No.13480067).

\section{References}

[1] Nambara, E.H. and Nonogaki (2012) Seed Biology in the 21st Century: Perspectives and New Directions. Plant \&Cell Physioloy, 53, 1-4. https://doi.org/10.1093/pcp/pcr184

[2] Dickson, M.H. (1980) Genetic Aspects of Seed Quality. Hortscience, 15, 771-774. https://www.cabdirect.org/cabdirect/abstract/19811602489

[3] Hilhorst, H.W., Finch-Savage, W.E., Buitink, J., et al. (2010) Dormancy in Plant Seeds: Dormancy and Resistance in Harsh Environments. Springer, Berlin, 43-67. https://doi.org/10.1007/978-3-642-12422-8_4

[4] Kazmi, R.H., Khan, N. and Willems, L.A. (2006) JoinMap 4, Software for the Calculation of Genetic Linkage Maps Inexperimental Populations. Wageningen, Netherlands.

[5] Gupta, M.L., George, D.L. and Parwata, I.G. (2005) Effect of Harvest Time and Drying on Super Sweet Corn Seed Quality. Seed Science \& Technology, 33, 167-176. https://doi.org/10.15258/sst.2005.33.1.17

[6] Rodo, A.B. and Marcos, F.J. (2003) Onion Seed Vigor in Relation to Plant Growth and Yield. Horticultura Brasileira, 31, 220-222. https://doi.org/10.1590/S0102-05362003000200020

[7] Bentsink, L., Alonso-Blanco, C., Vreugdenhil, D., et al. (2000) Genetic Analysis of Seed-Soluble Oligosaccharides in Relation to Seed Storability of Arabidopsis. Plant Physiology, 124, 1595-1604. https://doi.org/10.1104/pp.124.4.1595

[8] Ellis, R, H. and Roberts, E.H. (1981) The Quantification of Aging and Survival in Orthodox Seeds. Seed Science and Technology, 9, 373-409.

[9] Delouche, J.C. and Baskin, C.C. (2016) Accelerated Aging Techniques for Predicting the Relative Storability of Seed Lots. Seed Science and Technology, 1, 427-452.

[10] Hampton, J.G. and TeKrony, D.M. (1995) Handbook of Vigour Test Methods. 3rd Edition, The International Seed Testing Association, Zurich.

[11] Powell, A.A. and Matthews, S. (2005) Towards the Validation of the Controlled Deterioration, a New Vigour Test for Small Seeded Vegetables. Seed Testing International, 129, 21-24.

[12] Bewley, J.D. and Black, M. (1994) Seeds: Physiology of Development and Germination, 38, 1551.

[13] Clerkx, E.J., Blankestijn-De Vries, H., Ruys, G.J., et al. (2004a) Genetic Differences 
in Seed Longevity of Various Arabidopsis mutants. Physiology Plant, 121, 448-461. https://doi.org/10.1111/j.0031-9317.2004.00339.x

[14] Miura, K., Lyn, S.Y., Yano, M. and Nagamine, T. (2002) Mapping Quantitative Trait Loci Controlling Seed Longevity in Rice (Oryza sativa L.). Theoretical and Applied Genetic, 104, 981-986. https://doi.org/10.1007/s00122-002-0872-X

[15] Contreras, S. Bennett, M.A. and Metzger, J.D. (2009) Red to Far-Red Ratio during Seed Development Affects Lettuce Seed Germinability and Longevity. HortScience, 44, 130-134.

[16] Zhu, C., Gore, M., Buckler, E.S., et al. (2008) Status and Prospects of Association Mapping in Plants. Plant Genome, 1, 5-20. https://doi.org/10.3835/plantgenome2008.02.0089

[17] Zeng, D.L., Guo, L.B., Xu, Y.B., et al. (2006) QTL Analysis of Seed Storability in Rice. Plant Breeding, 125, 57-60. https://doi.org/10.1111/j.1439-0523.2006.01169.x

[18] Xue, Y., Zhang, S.Q., Yao, Q.H., et al. (2008) Identification of Quantitative Trait Loci for Seed Storability in Rice (Oryza sativa L.). Euphytica, 164, 739-744.

https://link.springer.com/article/10.1007\%2Fs10681-008-9696-3 https://doi.org/10.1007/s10681-008-9696-3

[19] Nagel, M., Vogel, H., Landjeva, S., et al. (2009) Seed Conservation in ex Situ Genebanks Genetic Studies on Longevity in Barley. Euphytica, 170, 5-14. https://doi.org/10.1007/s10681-009-9975-7

[20] Nagel, M., Rosenhauer, M., Willner, E., et al. (2011) Seed Longevity in Oilseed Rape (Brassica napus L.) Genetic Variation and QTL Mapping. Plant Genetic Research, 9, 260-263. https://doi.org/10.1017/S1479262111000372

[21] Rehman Arif, M.A., Nagel, M., Neumann, K., et al. (2012) Genetic Studies of Seed Longevity in Hexaploid Wheat using Segregation and Association Mapping Approaches. Euphytica, 186, 1-13. https://doi.org/10.1007/s10681-011-0471-5 https://link.springer.com/article/10.1007\%2Fs10681-011-0471-5

[22] Jannink, J.L., Blink, M.C. and Jansen, R.C. (2001) Using Complex Pedigrees to Map Valuable Genes. Trends Plant Science, 6, 337-342. https://doi.org/10.1016/S1360-1385(01)02017-9

[23] Rajjou, L., Lovigny, Y., Groot, S.P., et al. (2008) Proteome-Wide Characterization of Seed Aging in Arabidopsis: A Comparison between Artificial and Natural Aging Protocols. Plant Physiology, 148, 620-641. https://doi.org/10.1104/pp.108.123141

[24] Xin, X., Lin, X.H., Zhou, Y.C., et al. (2011) Proteome Analysis of Maize Seeds: The Effect of Artificial Aging. Physiologia Plantarum, 143, 126-138. https://doi.org/10.1111/j.1399-3054.2011.01497.x

[25] Wu, X.L., Liu, H.Y., Wang, W., et al. (2011) Proteomic Analysis of Seed Viability in Maize. Acta Physiologiae Plantarum, 33, 181-191. https://link.springer.com/article/10.1007\%2Fs11738-010-0536-4 https://doi.org/10.1007/s11738-010-0536-4

[26] Zeng, D.L., Qian, Q., Yasukumi, K., et al. (2002) Study on Storability and Morphological Index in Rice (Oryza sativa L.) under Artificial Aging. Acta Agronomica Sinica, 28, 551-554.

[27] Lander, E.S., Green, P., Abrahamson, J., et al. (1987) MAPMAKER: An Interactive Computer Package for Constructing Primary Genetic Linkage Maps of Experimental and Natural Populations. Genomics, 1, 174-181.

https://www.sciencedirect.com/science/article/pii/0888754387900103 
https://doi.org/10.1016/0888-7543(87)90010-3

[28] Han, Z., Ku, L., Zhang, Z., et al. (2014) QTLs for Seed Vigor-Related Traits Identified in Maize Seeds Germinated under Artificial Aging Conditions. PLoS ONE, 9, e92535. http://journals.plos.org/plosone/article?id=10.1371/journal.pone.0092535 https://doi.org/10.1371/journal.pone.0092535

[29] Zeng, Z.B. (1994) Precision Mapping of Quantitative Trait Loci. Genetics, 136, 1457-1468.

[30] Trachsel, S., Messmer, R., Stamp, P., et al. (2009) Mapping of QTLs for Lateral and Axile Root Growth of Tropical Maize. Theoretical and Applied Genetic, 119, 1413-1424. https://link.springer.com/article/10.1007/s00122-009-1144-9 https://doi.org/10.1007/s00122-009-1144-9

[31] Churchill, G.A. and Doerge, R.W. (19986) Empirical Threshold Values for Quantitative Trait Mapping. Genetics, 142, 285-294. https://www.ncbi.nlm.nih.gov/pubmed/7851788

[32] Lynch, M. and Walsh, B. (1998) Genetics and Analysis of Quantitative Traits.

[33] Arcade, A., Labourdette, A., Falque, M., et al. (2004) BioMercator: Integrating Genetic Maps and QTL towards Discovery of Candidate Genes. Bioinformatics, 20 , 2324-2326. https://academic.oup.com/bioinformatics/article/20/14/2324/214029 https://doi.org/10.1093/bioinformatics/bth230

[34] Chardon, F.B., Virlon, L., Moreau, M., et al. (2004) Genetic Architecture of Flowering Time in Maize as Inferred from Quantitative Trait Loci Meta-Analysis and Synteny Conservation with Rice Genome. Genetics, 168, 2169-2185. https://doi.org/10.1534/genetics.104.032375

[35] Hirotugu, A. (1974) A New Look at the Statistical Model Identification. IEEE Transactions on Automatic Control, 19, 716-723. https://doi.org/10.1109/TAC.1974.1100705

[36] Goffinet, B. and Gerber, S. (2000) Quantitative Trait Loci: A Meta-Analysis. Genetics, 155, 463-473.

http://med.wanfangdata.com.cn/Paper/Detail?id=PeriodicalPaper_JJ0215111768

[37] Walters, C. (1998) Understanding the Mechanisms and Kinetics of Seed Aging. Seed Science Research, 8, 223-244. https://doi.org/10.1017/S096025850000413X

[38] Rajjou, L. and Debeaujon, I. (2008) Seed Longevity: Survival and Maintenance of High Germination Ability of Dry Seeds. C. R. Biologies, 331, 796-805. https://doi.org/10.1016/j.crvi.2008.07.021

[39] Priestley, D.A. (1986) Seed Aging: Implications for Seed Storage and Persistence in the Soil. Comstock, Ithaca London.

[40] Smith, M.T. and Berjak, P. (1995) Deteriorative Changes Associated with the Loss of Viability of Stored Desiccation-Tolerant and Desiccation-Sensitive Seeds.

[41] McDonald, M.B. (1999) Seed Deterioration: Physiology, Repair and Assessment. Seed Science Technology, 27, 177-237. http://europepmc.org/abstract/agr/ind21988950

[42] Decourcelle, M., Perez-Fons, L., Baulande, S., et al. (2015) Combined Transcript, Proteome, and Metabolite Analysis of Transgenic Maize Seeds Engineered for Enhanced Carotenoid Synthesis Reveals Pleotropic Effects in Core Metabolism. Journal of Experimental Botany, 66, 3141-3150. https://www.ncbi.nlm.nih.gov/pubmed/25796085

[43] Hyndman, D., Bauman, D.R. and Heredia, V.V. (2003) The aldo/keto Reductase 
Superfamily Homepage. Chemico-Biological Interactions, 143-144, 621-631. https://www.ncbi.nlm.nih.gov/pubmed/12604248?dopt=Abstract

[44] Kim, S.T., Wang, Y., Kang, S.Y., et al. (2009) Developing Rice Embryo Proteomics Reveals Essential Role for Embryonic Proteins in Regulation of Seed Germination. Journal of Proteome Research, 8, 3598-3605. https://doi.org/10.1021/pr900358s

[45] Lemmon, K.A. and Schlessinger, J. (2010) Cell Signaling by Receptor Tyrosine Kinases. Cell, 141, 1117-1134. https://doi.org/10.1016/j.cell.2010.06.011

[46] Stone, J.M. and Walker, J.C. (1995) Plant Protein Kinase Families and Signal Transduction. Plant Physiology, 106, 451-457. https://doi.org/10.1104/pp.108.2.451 https://www.ncbi.nlm.nih.gov/pubmed/7610156

[47] Cabiscol, E., Piulats, E., Echave, P., et al. (2000) Oxidative Stress Promotes Specific Protein Damage in Saccharomyces cerevisiae. Journal of Biology Chemistry, 275, 27393-27398. https://doi.org/10.1074/jbc.M003140200

[48] Dell'Aquila (1994) Wheat Seed Aging and Embryo Protein Degradation. Seed Science Research, 4, 293-298. https://doi.org/10.1017/S0960258500002324

[49] Ching, T.M. and Schoolcraft, I. (1968) Physiological and Chemical Differences in Aged Seeds. Crop Science, 8, 407-409.

https://eurekamag.com/research/014/605/014605144.php https://doi.org/10.2135/cropsci1968.0011183X000800040003x

[50] Kocsy, G., Von Ballmoos, P., Ruegsegger, A., et al. (2001) Increasing the Glutathione Content in a Chilling-Sensitive Maize Genotype using Safeners Increased Protection against Chilling-Induced Injury. Plant Physiology, 127, 1147-1156. http://www.jstor.org/stable/4280172 https://doi.org/10.1104/pp.010107

[51] Sattler, S.E., Gilliland, L.U., Magallanes-Lundback, M., et al. (2004) Vitamin E Is Essential for Seed Longevity and for Preventing Lipid Peroxidation during Germination. Plant Cell, 16, 1419-1432. https://www.ncbi.nlm.nih.gov/pubmed/15155886 https://doi.org/10.1105/tpc.021360

[52] Job, C., Rajjou, L., Lovigny, Y., et al. (2005) Patterns of Protein Oxidation in Arabidopsis Seeds and during Germination. Plant Physiology, 138, 790-802.

http://europepmc.org/abstract/MED/15908592 https://doi.org/10.1104/pp.105.062778

[53] Kibinza, S., Vinel, D., Come, D., et al. (2006) Sunflower Seed Deterioration as Related to Moisture Content during Aging, Energy Metabolism and Active Oxygen Species Scavenging. Physiology Plant, 128, 496-506.

https://doi.org/10.1111/j.1399-3054.2006.00771.x

[54] Bingham, I.J. and Merritt, G.J. (1999) Effect of Seed Aging on Early Post-Germination Root Extension in Maize: A Spatial and Histological Analysis of the Growth-Zone. Seed Science \& Technology, 27, 151-162. https://eurekamag.com/research/010/563/010563017.php 